\title{
Temporal integration shown in the early and late components of the human blink reflex
}

\author{
GEOFFREY R. HAMMOND and STUART DENNON \\ University of Western Australia, Nedlands, Australia
}

\begin{abstract}
The effect of a second stimulus presented after the reflex elicitor on the two successive EMG components of the cutaneous blink reflex in normal human subjects was examined. An acoustic stimulus presented up to $6 \mathrm{msec}$ after the eliciting stimulus had no effect on size or latency of the first component recorded ipsilateral to the eliciting stimulus (R1), but the size of the second component recorded contralateral to the eliciting stimulus (CR2) was increased and its latency decreased. Enhancement of CR2 was independent of the stimulus onset asynchrony (SOA) of the two stimuli and was an increasing function of the intensity of the acoustic stimulus. CR2 enhancement was present when the second stimulus was by itself below threshold for reflex elicitation. A second percutaneous stimulus that appeared up to $8 \mathrm{msec}$ after the eliciting stimulus increased the size of R1 at all SOAs and increased its latency at the longest SOA tested. CR2 amplitude was unaffected and there was only a small effect on CR2 latency. The components of the blink reflex are not determined fully at the time of reflex elicitation, but are sensitive to low-level stimuli that occur in the reflex latency. This sensitivity was restricted to percutaneous stimuli for R1, indicating that acoustic stimuli do not have direct access to the $R 1$ pathway. The results show an inverse relationship between the size of $\mathrm{R} 1$ and the size of CR2, which may be a compensatory reduction in CR2 following closure of the eyelid initiated by large R1 components.
\end{abstract}

The human blink reflex is useful as a diagnostic tool in neurology (Kimura, 1973; Sazbon, Solzi, Steinvil, \& Becker, 1988; Schmalohr \& Linke, 1988) and as a model system for studying neural function. It has been used to study motor learning (Evinger \& Manning, 1988) and the integration of stimulus energy distributed over time (Blumenthal \& Berg, 1986; Plant \& Hammond, 1989). The percutaneously elicited blink reflex is especially useful as a model system. It has two components that can be recorded electromyographically from the orbicularis oculi muscle, a brief short-latency component mediated by an oligosynaptic pathway (R1), and a more prolonged later component (R2) mediated by a polysynaptic pathway. $R 1$ is found ipsilateral to the eliciting stimulus, whereas $\mathbf{R} 2$ occurs bilaterally (the $\mathbf{R} 2$ components ipsilateral and contralateral to the eliciting stimulus are denoted IR2 and CR2, respectively). The pathways differ in the complexity of their central organization: the R1 pathway appears to have only one interneuron between the afferent and efferent limbs, whereas the $R 2$ pathway is complex and includes bilateral structures in the brainstem reticular formation (see, e.g., Ongerboer de Visser \& Kuypers, 1978; Schmalohr \& Linke, 1988). The size and the latency of the two successive electromyographic (EMG) components reflect the excitability of their different brainstem pathways.

Temporal integration of stimulus energy has been shown by larger and faster blinks in response to successive

Send correspondence to Geoff Hammond, Department of Psychology, University of Western Australia, Nedlands, WA 6009, AUSTRALIA (e-mail: jeff@psy.uwa.oz.au). closely spaced blink-eliciting stimuli as opposed to a single blink-eliciting stimulus alone (Blumenthal \& Berg, 1986; Plant \& Hammond, 1989). To date, temporal integration has been shown only for the $\mathrm{R} 2$ component of the percutaneously elicited blink reflex and for the longlatency EMG component of the acoustically elicited blink, which is analogous to the R2 component of the cutaneous reflex. Blumenthal and Berg (1986) found that reflexes elicited by pairs of 3-msec acoustic pulses with stimulus onset asynchronies (SOAs) of up to about $40 \mathrm{msec}$ were larger than control reflexes elicited by single 3-msec pulses. This result was replicated by Plant and Hammond (1989), who found that the larger reflexes with paired stimuli also had shorter latencies than the control reflexes did. Temporal integration is not restricted to pairs of acoustic stimuli. Augmentation of R2 has been found with pairs of percutaneous stimuli (brief electrical pulses delivered to the skin over the supraorbital branch of the trigeminal nerve) and with cross-modal stimulus pairs (acoustic-percutaneous and percutaneous-acoustic pairs; Plant \& Hammond, 1989). In all cases, integration is present with paired stimuli presented with SOAs of up to about $40 \mathrm{msec}$.

Temporal integration shown in reflex size is not a purely sensory phenomenon; as noted above, it occurs with each member of the stimulus pair from a different modality, and the time course of integration of percutaneous stimuli is not different from that of acoustic stimuli, although psychophysical work shows different time parameters for sensory integration in these two modalities (Scharf \& Buus, 1986; Scharf \& Houtsma, 1986; Sherrick \& Cholewiak, 1986). Integration shown in reflex expression therefore 
occurs at least in part outside of specific sensory pathways and measures a different aspect of sensory processing from that measured by psychophysical judgments. Plant and Hammond (1989) suggested that integration shown in the acoustic blink reflex and the $\mathbf{R} 2$ component of the cutaneous blink reflex results from summation of activity within the non-sensory-specific structures in the lower brainstem reticular formation, which are known to integrate input from different sensory modalities. This proposal can be easily tested. If temporal integration is only a property of the reflex-organizing structures in the brainstem reticular formation, then stimulus pairs with an appropriately short SOA will augment the cutaneous R2 component, but $R 1$, whose pathway does not include these structures, will be unaffected. If temporal integration is found in $\mathrm{R} 1$, it will show that temporal integration shown in reflex size is a property of relatively simple neural pathways.

A second question asked in the present research is whether the second member of a stimulus pair has to be a reflex elicitor in order for integration to occur. Previous studies of temporal integration in the human blink reflex have been restricted to pairs of successive reflexogenic stimuli, and it is not known whether temporal integration occurs when the second member of a stimulus pair is below the threshold for reflex elicitation when presented alone. It has been found recently (Hammond \& Leitner, 1990 ) that the acoustic startle reflex in rats is augmented by nonreflexogenic stimuli presented after the reflexeliciting stimulus. In the present experiments, we examined whether this occurs for the human blink reflex.

\section{EXPERIMENT 1}

\section{Method}

Subjects. Twelve healthy adult male volunteers were used as subjects. Their ages ranged from 21 to 45 years, with a median of 24 years.

Apparatus and Procedure. Cutaneous blink reflexes were elicited by 0.5 -msec biphasic square-wave pulses delivered by a Grass SD 9 stimulator and $\mathrm{CCU}-1$ constant current unit. The stimuli were presented through small (5-mm-diameter) stainless steel electrodes attached to the skin on the right side of the subject's forehead, overlying the supraorbital branch of the trigeminal nerve. The electrodes were separated by $2 \mathrm{~cm}$. The intensity threshold for elicitation of R1 for each subject was determined by presenting single stimuli at intervals of $20-40 \mathrm{sec}$, beginning at an intensity of about $0.5 \mathrm{~mA}$ and increasing in steps of about $0.2 \mathrm{~mA}$ until an R1 was evident on the oscilloscope trace of the EMG activity on three successive stimulus presentations. The $\mathrm{R} 1$ elicitation thresholds ranged from 3.2 to $35.0 \mathrm{~mA}$ with a median of $12.5 \mathrm{~mA}$.

Acoustic stimuli were clicks formed by delivering a 3-msec square wave to headphones worn by the subject. The intensity threshold for elicitation of the acoustic blink reflex was determined in a manner similar to the determination of the cutaneous R1 intensity threshold: clicks were delivered at 20- to 40-sec intervals, beginning at about $60 \mathrm{~dB}(\mathrm{~A}) \mathrm{SL}$ and increasing in steps of about $5 \mathrm{~dB}$ until reflex activity was evident on three successive stimulus presentations. Acoustic intensities were estimated with a Brüel and Kjaer artificial ear. First, it was established that the headphone drivers transduced the 3-msec click into a near-sinusoidal stimulus with a frequency of $156 \mathrm{~Hz}$. Second, the RMS sound-pressure levels of continuous tones at this frequency were determined, and these levels are reported as the sound-pressure equivalents of the brief stimuli used. The thresholds obtained ranged from 64 to $105 \mathrm{~dB}(\mathrm{~A}) \mathrm{SL}$ with a median of $95 \mathrm{~dB}(\mathrm{~A}) \mathrm{SL}$.

EMG activity was recorded by Grass gold-plated surface recording electrodes attached bilaterally to the skin overlying the orbicularis oculi muscle. One electrode was located on the lower lid margin in line with the pupil as the subject looked straight ahead, and the other was located on the canthus, about $2 \mathrm{~cm}$ lateral to the first. A ground electrode was placed on the left mastoid process, contralateral to the stimulating electrodes. The EMG signals were amplified with Grass 7P511 amplifiers with half-amplitude high-pass and low-pass frequency settings on $3 \mathrm{~Hz}$ and $30 \mathrm{kHz}$, respectively. The gain was adjusted so that vigorous blinks produced signals within a 5-V range, the limit of the analog-to-digital converters.

The subjects sat quietly in a sound-attenuated room, with their eyes closed lightly. Reflex blinks were elicited by the percutaneous stimulus presented alone (control trials) and with the percutaneous stimulus followed by an acoustic stimulus. Six experimental conditions were formed by the factorial combination of two SOAs ( 3 and $6 \mathrm{msec}$ ) and three levels of acoustic stimulus intensity (the acoustic blink reflex threshold intensity, the threshold intensity attenuated by $12 \mathrm{~dB}$, and the threshold intensity attenuated by $36 \mathrm{~dB}$ ). Sixteen control trials and 8 trials in each experimental condition were given, for a total of 64 trials. The sequence of trials was determined by the incomplete counterbalancing method described by D'Amato (1979), to control for sequence effects. Trials were given randomly at intervals of $16,18,20,22$, and $24 \mathrm{sec}$.

Data acquisition and analysis. The amplified EMG signals from each eye were digitized by 12-bit analog-to-digital converters at $2 \mathrm{kHz}$. Peak-to-peak amplitudes of R1 and both IR2 and CR2 were measured during the periods from 10-25 msec and 25-105 msec after the percutaneous stimulus, respectively. Median peak-to-peak amplitudes for each subject were later calculated for each component and experimental condition, and these median amplitudes were then expressed as a ratio of the median control amplitude. Logarithms of the amplitude ratios were then taken, because the distribution of ratio scores is positively skewed. Geometric means of the amplitude ratios (the arithmetic means of the logarithms of the amplitude ratios) are reported. Latencies of the two components were scored trial-by-trial from the EMG trace stored on a digital oscilloscope. Analyses of variance of $\log$ amplitude ratios and latencies were done, and the proportion of variance accounted for by significant effects ( $\omega^{2}$; Hays, 1963) is reported for statistically reliable effects. Because all designs had fully repeated measures, $\omega^{2}$ was calculated with the sum of squares for subjects excluded from the total sum of squares. The values are therefore not affected by the heterogeneity of the particular subjects sampled in an experiment. Two-tailed Dunnett's $t$ tests were used to test whether mean $\log$ amplitude ratios from particular experimental conditions differed reliably from zero (the control level, corresponding to an amplitude ratio of 1.0 ) and whether mean latencies differed reliably from mean latency on control trials.

\section{Results}

Correlation of the median amplitudes and mean latencies from each subject showed that IR2 and CR2 were strongly associated $(r=0.88$ and 0.83 for amplitude and latency, respectively). Because of this dependency, only results for CR2 will be reported; any effects noted for CR2 were also present in IR2.

Blink amplitude. R1 amplitude was not affected by the presence of a trailing acoustic stimulus in any of the experimental conditions. The geometric means of the amplitude ratios in the different conditions ranged from -0.04 to 0.04 with a mean of $0.00(S E=0.02)$. Dunnett's 
$t$ tests showed that none of the means was significantly greater than the control level of zero, the logarithm of an amplitude ratio of 1.0. In contrast, CR2 amplitude was augmented in all of the experimental conditions. The geometric mean amplitude ratios from the different conditions ranged from 0.08 to 0.14 with a mean of 0.12 $(S E=0.02)$. The untransformed amplitude ratios ranged from 1.26 to 1.47 , with an overall mean of 1.39. Amplitude augmentation was similar in the different experimental conditions, and analysis of variance showed no effect of either the intensity of the trailing acoustic stimulus $[F(2,22)=1.93, p>.05]$ or the SOA $[F(1,11)=1.64$, $p>.05]$, nor was there an interaction between them $[F(2,22)=1.47, p>.05]$.

Blink latency. The mean latency of R1 was $12 \mathrm{msec}$ in the control condition and in each experimental condition in which the percutaneous stimulus was followed by an acoustic stimulus. CR2 latencies are shown in Figure 1. CR2 latency was a function of the intensity of the trailing acoustic stimulus, and it was progressively shorter with more intense acoustic stimuli. The effect of acoustic stimulus intensity was statistically reliable $[F(3,33)=8.64$, $p<.05]$ and accounted for $24 \%$ of the variance $\left(\omega^{2}=\right.$ $0.24)$. Dunnett's $t$ tests showed that the mean latencies from the conditions in which the acoustic stimulus was attenuated by 0 and $12 \mathrm{~dB}$ were both significantly shorter than control latency; latency from the condition in which the acoustic stimulus was attenuated by $36 \mathrm{~dB}$ was not significantly shorter than control latency. CR2 latency was also a function of the SOA, and it was shorter with the 3-msec as opposed to the 6-msec SOA; the SOA variable was statistically reliable $[F(1,11)=7.32, p<.05]$, but it accounted for only a small amount of the variance $\left(\omega^{2}=0.04\right)$. The interaction of acoustic stimulus intensity and SOA was not statistically reliable.

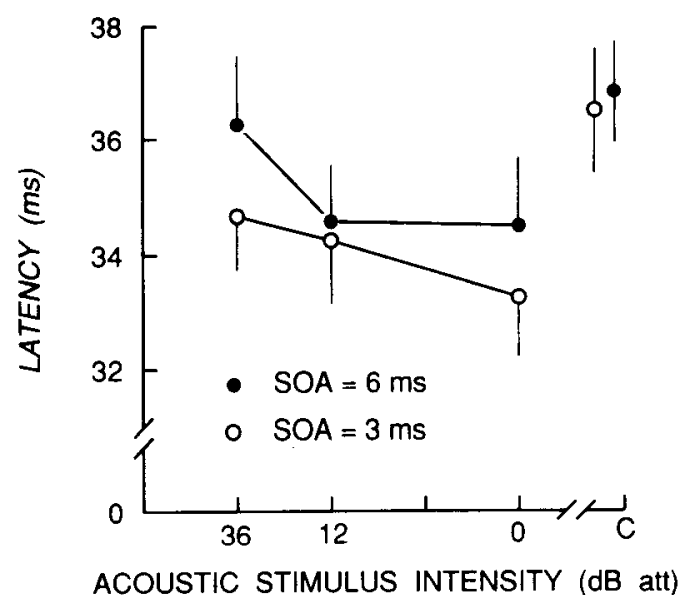

Figure 1. Mean CR2 latency at each of the three intensities of the acoustic stimulus and each of the two stimulus onset asynchronies in Experiment 1. Mean latency from each of the two sets of control trials (C) is shown at the right of the figure. Error bars show $1 S E$ for the experimental conditions and $\pm 1 S E$ for the control trials.

\section{Discussion}

The results of Experiment 1 are straightforward: The trailing acoustic stimulus enhanced CR2 (both amplitude augmentation and latency facilitation were present), but had no effect on either the amplitude or the latency of the $\mathrm{R} 1$ response. An acoustic stimulus that appeared in the response latency 3 or $6 \mathrm{msec}$ after an eliciting percutaneous stimulus increased the excitability of the pathway responsible for the $\mathrm{CR} 2$ response but did not affect the pathway responsible for the $\mathrm{R} 1$ response.

Enhancement of the CR2 response by a trailing acoustic stimulus replicates the previous report that stimulus energy is integrated across different stimulus modalities, although the effects on both amplitude and latency found here are generally smaller than those found in similar conditions in the earlier work (Plant \& Hammond, 1989). This difference may result from the more intense leading percutaneous reflex-eliciting stimuli used in the present experiment, in which the intensity was adjusted so that an R1 response was reliably elicited. The average intensity of the reflex-eliciting stimulus used here was about 3.5 times greater than that used by Plant and Hammond, which was set to the lower R2 elicitation threshold. The more intense leading stimulus used here would drive the circuitry more strongly, with less to be gained from the presentation of a trailing stimulus.

The observations extend Hammond and Leitner's (1990) finding that nonreflexogenic stimuli presented in the reflex latency augmented the acoustic startle reflex in rats. First, a similar augmentation is present in the CR2 component of the human blink reflex, showing it to be general across reflexes and species. Second, trailing stimuli can reduce reflex latency as well as augment reflex amplitude. This is the first demonstration that human reflexes are sensitive to stimuli that appear after the reflex elicitor and are by themselves below threshold for reflex elicitation. The protective function of larger and faster blinks is presumably enhanced, implying that the plasticity of reflex expression after elicitation is an important biological phenomenon.

Perhaps we did not find any effects on the R1 pathway because it is too simple to support integration of energy across modalities, or because the acoustic stimulus was delayed too long after the reflex-eliciting stimulus to affect the R1 pathway. These hypotheses were examined in Experiments 2 and 3.

\section{EXPERIMENT 2}

It was possible that we had failed to find temporal integration in the R1 component in Experiment 1 because the trailing acoustic stimulus was presented too late to be effective. In Experiment 2, we sought to determine whether or not temporal integration of paired percutaneous and acoustic stimuli was present in R1 when an acoustic stimulus appeared simultaneously with the eliciting percutaneous stimulus and at brief delays after it. 


\section{Method}

Subjects. Six healthy adult male volunteers were tested. Five had participated in Experiment 1. Their ages ranged from 21 to 45 years, with a median of 24 years.

Apparatus and Procedure. The apparatus and general experimental procedures were unchanged from those of Experiment 1. Subjects were presented with a percutaneous reflex-eliciting stimulus alone (control trials) and a percutaneous reflex-eliciting stimulus followed by an acoustic reflex-eliciting stimulus at SOAs of 0,1 , 2 , and 3 msec. The intensity of the leading percutaneous stimulus was adjusted for each subject as in Experiment 1 to produce a blink reflex with a reliable $R 1$ component. The $R 1$ threshold intensities ranged from 3.4 to $15.0 \mathrm{~mA}$, with a median of $11.2 \mathrm{~mA}$. The intensity of the acoustic stimulus was adjusted in a similar way to produce a reliable acoustic blink reflex. These thresholds ranged from 70 to $102 \mathrm{~dB}(\mathrm{~A}) \mathrm{SL}$, with a median of $82 \mathrm{~dB}$ (A) SL. These threshold intensities were used in the experimental trials. Twelve trials were given in each of the five conditions, for a total of 60 trials. The trials were sequenced according to the incomplete counterbalancing method described in the section on Experiment 1.

\section{Results}

CR2 and IR2 amplitudes and latencies were again correlated highly ( $r=0.92$ for both measures), and therefore only measures of CR2 amplitude and latency will be reported.

Blink amplitude. R1 amplitude did not vary from control amplitude in any condition. The geometric means of the amplitude ratios for $R 1$ ranged from -0.02 to 0.05 , with a mean of $0.02(S E=0.02)$. Dunnett's $t$ tests showed that none of these values was reliably greater than zero. As in the previous experiment, however, CR2 amplitude was augmented in the same conditions. The geometric means of the amplitude ratios for this component were similar in the different conditions; they ranged from 0.14 to 0.15 , with a mean of $0.14(S E=0.03)$. Dunnett's $t$ tests showed that the geometric mean amplitude ratio in each condition was greater than zero. The untransformed amplitude ratios ranged from 1.43 to 1.53 , with a mean of 1.49. There was no systematic effect of SOA on the amount of augmentation $(F<1)$.

Blink latency. There was no effect on mean R1 latency, which was $11 \mathrm{msec}$ in the control condition and in each experimental condition. CR2 latencies varied in the different experimental conditions. The mean control reflex latency was $36 \mathrm{msec}$, and the mean latencies with the $0-, 1-, 2-$, and 3-msec SOAs were $32,34,32$, and $33 \mathrm{msec}$, respectively. The effect of SOA was statistically reliable $[F(4,20)=6.90, p<.05]$, and it accounted for $49 \%$ of the variance $\left(\omega^{2}=0.49\right)$. Dunnett's $t$ tests showed that the CR2 latencies from all the paired-stimulus conditions except the 1-msec SOA were shorter than mean control latency.

\section{Discussion}

These results extend those of Experiment 1 and show that the Rl component of the cutaneous blink reflex is unaffected by an acoustic stimulus presented simultaneously with the percutaneous reflex-eliciting stimulus or up to $3 \mathrm{msec}$ after it. The conclusion from both experiments together is that whereas the CR2 component of the cutaneous blink reflex is enhanced by acoustic stimuli that occur simultaneously with the reflex-eliciting stimulus and up to at least $6 \mathrm{msec}$ after it, the $\mathrm{R} 1$ component is not. Either acoustic stimuli that appear in the response latency do not affect the excitability of the R1 pathway, or the $R 1$ pathway (unlike the more complex $R 2$ pathway) does not support temporal integration of stimulus energy.

\section{EXPERIMENT 3}

In Experiment 3, we determined whether R1 amplitude and latency would be affected by a second percutaneous stimulus that appeared after a leading percutaneous reflexeliciting stimulus.

\section{Method}

Subjects. Six healthy male volunteers were tested. Three had participated in Experiments 1 and 2. Their ages ranged from 21 to 28 years with a median of 24.5 years.

Apparatus and Procedure. The apparatus and general experimental procedures were similar to those of the first two experiments. A second Grass SD9 stimulator and CCU-1 constant current unit was added to deliver the second percutaneous stimulus. The subjects were presented with a percutaneous reflex-eliciting stimulus alone (control trials) and with a percutaneous reflex-eliciting stimulus followed after $1,2,4$, and $8 \mathrm{msec}$ by a second percutaneous stimulus at one of two intensity levels.

The intensity of the leading percutaneous stimulus was adjusted for each subject as in the previous experiments, to produce a blink reflex with a reliable $R 1$ component. The $R 1$ threshold intensities ranged from 3.0 to $8.5 \mathrm{~mA}$ with a median of $4.6 \mathrm{~mA}$. The second percutaneous stimulus was presented at the R1 threshold level (the same intensity as the leading stimulus) or at the Rl threshold intensity attenuated by $3 \mathrm{~dB}$. Intensities of the second percutaneous stimulus ranged from 2.1 to $6.0 \mathrm{~mA}$ with a median of $3.2 \mathrm{~mA}$. Six trials were given in each of the eight conditions (two intensities of the trailing stimulus at each of four SOAs) together with 12 control trials for a total of 60 trials. Trials were sequenced according to the incomplete counterbalancing method described in Experiment 1.

\section{Results}

CR2 and IR2 amplitudes and latencies were again correlated ( $r=0.76$ for both measures).

Blink amplitude. R1 amplitude was strongly augmented by the trailing percutaneous stimulus. Geometric means of R1 amplitude ratios are shown in Figure 2. Augmentation was greater with the more intense trailing stimulus $[F(1,5)=6.68, p<.05]$; this variable accounted for $34 \%$ of the variance. The effect of SOA was also statistically reliable $[F(3,15)=4.85, p<.05]$, although it accounted for only $6 \%$ of the variance. Dunnett's $t$ tests showed that the mean amplitude ratio at the $1-\mathrm{msec}$ SOA was significantly smaller than at the 2- and 4-msec SOAs but not the $8-\mathrm{msec}$ SOA. The interaction between intensity of the second stimulus and SOA was not statistically reliable. Each mean amplitude was shown by Dunnett's $t$ test to be reliably greater than zero. The untransformed amplitude ratios ranged from 3.45 to 7.45 in the different conditions, with a mean of 5.80 .

In contrast to the observations of $R 1$ in this experiment, and in contrast to the observations of CR2 in the previ- 


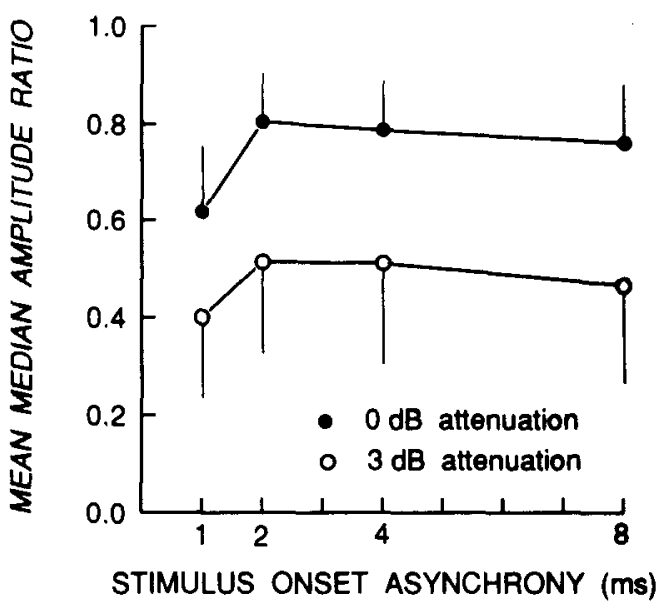

Figure 2. Geometric means of the median proportional R1 amplitudes at each of the four stimulus onset asynchronies and each of the two intensities of the second percutaneous stimulus in Experiment 3. Error bars show 1 SE.

ous experiments, CR2 was not augmented. The geometric mean amplitude ratios ranged from -.10 to .09 in the different conditions, with a mean of $.01(S E=0.02)$. Neither the effect of intensity of the second percutaneous stimulus $[F(1,5)=6.02, p>.05]$ nor the effect of SOA $[F(3,15)=1.16, p>.05]$ was significant. Dunnett's $t$ tests showed that none of the means of the experimental conditions was reliably different from zero.

Blink latency. Mean R1 latencies from the different conditions are shown in Figure 3. Latency was unaffected by the intensity of the second percutaneous stimulus $[F(1,5)=1.04, p>.05]$ but increased with SOA $[F(4,20)=7.28, p<.05]$. The SOA accounted for $43 \%$ of the variance.

Dunnett's $t$ tests showed that the latencies from the 8-msec SOA were reliably greater than control. The nature of the increase in R1 latency caused by the trailing stimulus can be seen in the frequency distributions of reflex latency from control trials and from the different SOAs, which are shown in Figure 4. The distribution of reflex latencies was not affected by the intensity of the trailing stimulus, so latencies from these conditions were combined.

The distribution of latencies from the 1- and 2-msec SOA conditions were similar to the distribution of the latencies from the control condition. At the 4- and 8-msec SOAs, however, the distribution of latencies was bimodal, with modes at 11 and $15 \mathrm{msec}$ at the 4-msec SOA and 11 and $19 \mathrm{msec}$ at the 8-msec SOA. The lengthening of the mean latency was therefore not the result of a continuous shift in latency, but of the emergence of a delayed R1 on some trials. R1 appeared sometimes at its typical control latency (about $11 \mathrm{msec}$ ), and sometimes with an additional delay equal to the SOA on that trial. This was seen in individual subjects, who showed both normal-latency and delayedlatency $R 1$ components on different trials.

R1 latencies were scored as "delayed" if they were $14 \mathrm{msec}$ or longer in the 4 -msec SOA conditions or
$18 \mathrm{msec}$ or longer in the 8-msec SOA conditions. This resulted in a proportion of 0.34 of all $R 1$ components being classified as delayed. The proportions of delayed trials did not vary appreciably with SOA (the proportions were 0.32 and 0.36 for the 4 - and 8-msec SOAs, respectively) or with intensity of the second stimulus $(0.28$ and 0.40 for the low- and high-intensity, respectively). Neither of these two factors nor their interaction was statistically reliable [SOA: $F(1,5)<1$; intensity of the second stimulus: $F(1,5)=1.23, p>.05$; interaction: $F<1]$. The

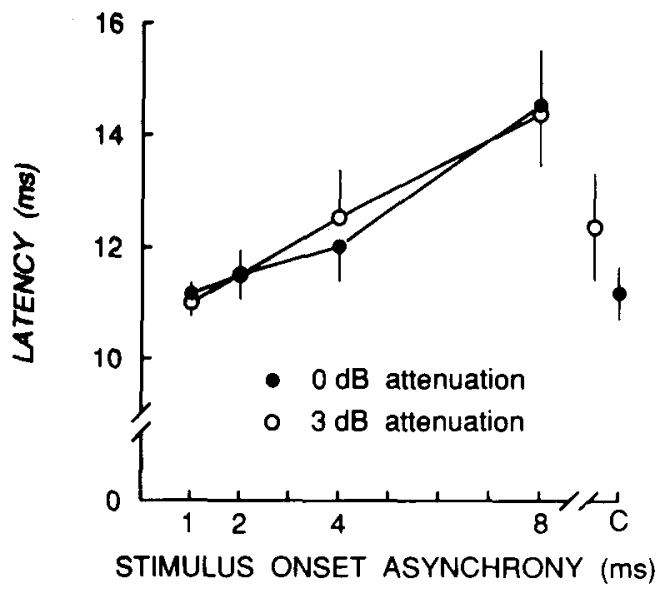

Figure 3. Mean R1 latency at each of the four stimulus onset asynchronies and each of the two intensities of the second percutaneous stimulus in Experiment 3. Mean latency from each of the two sets of control trials $(C)$ is shown at the right of the figure. Error bars show $1 S E$ for the experimental conditions and $\pm 1 S E$ for the control trials.
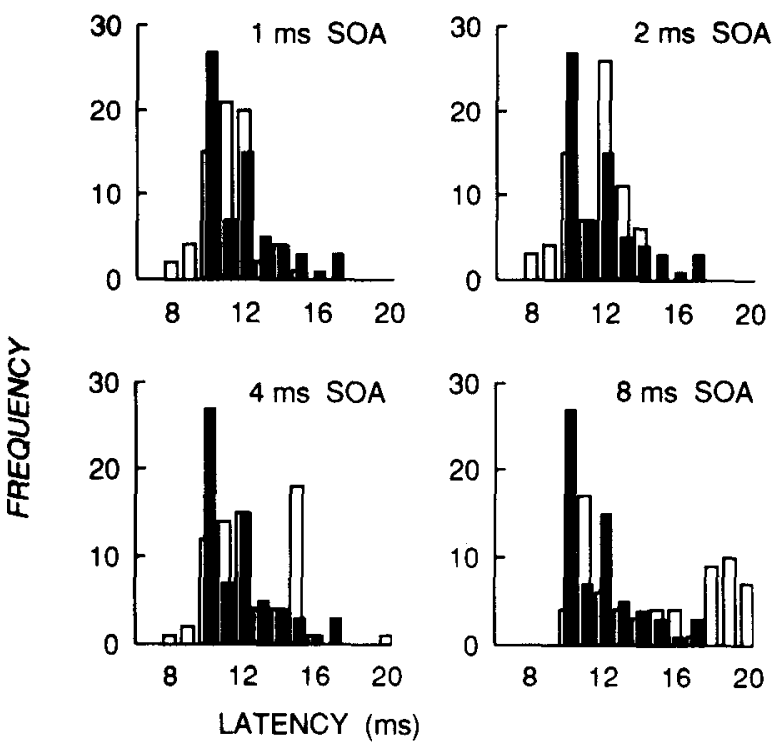

Figure 4. Frequency distributions of $\mathbf{R 1}$ latencies from control trials (solid bars) and from trials with a second percutaneous stimulus (open bars) at each of the four stimulus onset asynchronies in Experiment 3. The distribution of latencies from control trials is repeated in each panel to allow comparison with the distribution of latencies from trials with a second percutaneous stimulus. 
latency shift did not appear to affect reflex amplitude. Median amplitudes were calculated for normal- and delayedlatency $\mathrm{R} 1$ components for each subject. In conditions in which the median was based on at least two observations, the amplitude of the normal-latency $R 1$ was greater than that of the delayed-latency R1 in 6 out of 11 comparisons and was smaller in the other 5 comparisons. The shift in R1 latency similarly did not affect the latency of the subsequent IR2 component. In 16 cases in which a comparison of means based on two or more latencies could be made, the mean latency of IR2 components that followed a delayed-latency $R 1$ was longer than the mean latency of IR2 components that followed a normal-latency $R 1$ in 6 cases, shorter in 8 cases, and the same in the 2 remaining cases.

CR2 latency did not vary much between the different conditions. Mean latencies are shown in Figure 5. There was a statistically reliable effect of SOA $[F(4,20)=5.04$, $p<.05]$, which accounted for 23\% of the variance. Dunnett's $t$ tests showed that only the latency with the more intense second stimulus at the 2-msec SOA was reliably different from control latency. The intensity of the second stimulus was not effective $[F(1,5)=1.32, p>.05]$, but it did interact with $\operatorname{SOA}[F(4,20)=3.37, p<.05]$. This interaction accounted for $10 \%$ of the variance.

\section{Discussion}

R1 amplitude was increased markedly by a second percutaneous stimulus, showing that temporal integration does occur in relatively simple reflex pathways. Plant and Hammond's (1989) supposition that integration of stimulus energy occurs only in the reticular pathways is incorrect. Augmentation of R1 was an increasing function of the intensity of the second stimulus, and it was evident when the second stimulus was by itself below threshold

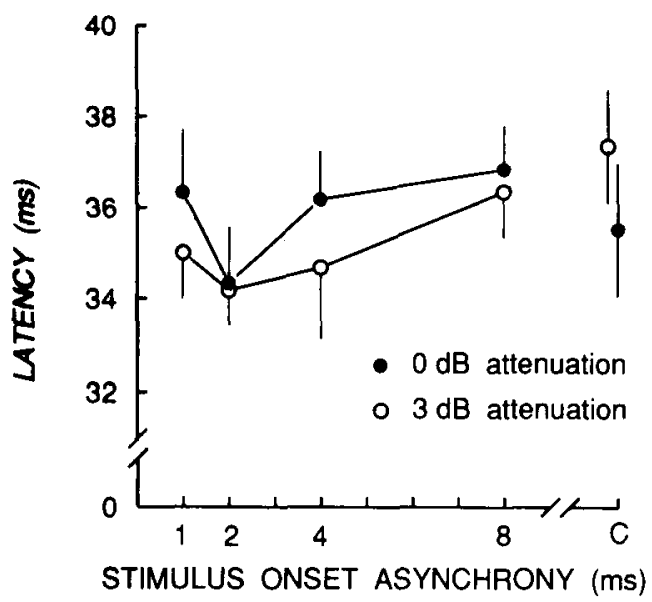

Figure 5. Mean CR2 latency at each of the four stimulus onset asynchronies and each of the two intensities of the second percutaneous stimulus in Experiment 3. Mean latency from each of the two sets of control trials (C) is shown at the right of the figure. Error bars show $1 S E$ for the experimental conditions and $\pm 1 S E$ for the control trials. for elicitation of R1. Augmentation of R1, however, was not accompanied by latency facilitation. Instead, $R 1$ latency was delayed on about one third of the 4- and 8-msec SOA trials by an amount about equal to the SOA, suggesting that the R1 was elicited by the second stimulus, not the first. Some of these instances can be accounted for by threshold fluctuations, which resulted in occasional failure of the first stimulus to elicit an R1. R1 was absent on about $8 \%$ of the control trials, in which only a single stimulus was presented. It may be that in the remaining cases, the second stimulus interrupted the activity initiated by the leading stimulus (which would otherwise have led to an observable $R 1$ ) and itself initiated activity that resulted in an observable $R 1$ after the usual latency of about $11 \mathrm{msec}$. In all instances, the amplitude of the delayed R1 was augmented above control levels, showing that the activity evoked by the leading stimulus, although not leading to reflex elicitation, did contribute to the size of the eventual reflex. The absence of any effect on CR2 amplitude was unexpected, because this component is augmented with percutaneous stimulus pairs in which the leading stimulus elicits R2, but is below threshold for R1 elicitation (Plant \& Hammond, 1989). Augmentation of R1 might suppress augmentation of the later CR2 (see the General Discussion).

\section{GENERAL DISCUSSION}

Temporal integration is evident in the $\mathrm{R} 1$ component of the blink reflex in specific conditions: $R 1$ amplitude is augmented with percutaneous-percutaneous stimulus pairs, but not with percutaneous-acoustic stimulus pairs. The excitability of the $R 1$ pathway is affected by a percutaneous stimulus presented after R1 elicitation, but is not affected by a similarly presented acoustic stimulus. This demonstrates that temporal integration of stimulus energy does occur in the relatively simple $R 1$ pathway, but that the auditory system does not have direct (and therefore quick) input to the R1 pathway. This is not a general effect of slow processing in the auditory system; brainstem auditory evoked responses show stimulus-related activity in the brainstem 3-4 msec after the stimulus (Jewett $\&$ Williston, 1971). The auditory system does have indirect input to the R1 pathways, shown by the effect of leading acoustic stimuli on R1 amplitude (Sanes \& Ison, 1979). This effect takes time to develop; it is evident with an SOA of $50 \mathrm{msec}$ but not with an SOA of $5 \mathrm{msec}$. This delay indicates that auditory effects on $\mathrm{R} 1$ excitability are mediated by longer and less direct pathways.

The present experiments revealed no decay of reflex augmentation with prolongation of SOA up to $8 \mathrm{msec}$. The only effect of SOA found was in Experiment 3, where the size of R1 was SOA-dependent. In this case, augmentation was less at the shortest SOA $(1 \mathrm{msec})$ than at the three longer SOAs tested $(2,4$, and $8 \mathrm{msec})$. This time course may indicate recovery from refractory effects present at the shortest SOA, an explanation proposed first by Marsh, Hoffman, and Stitt (1973) to account for similar 
effects of paired stimuli on whole-body acoustic startle in the rat. The persistence of augmentation without decay over the intervals tested suggests that it is unlikely to be the result of a subliminal fringe (a set of cells partially activated by the first stimulus recruited into discharge by the second), whose effects decay exponentially over time (see, e.g., Henneman, 1974). The mechanism of blink-reflex augmentation appears to be able to sustain activity without loss over at least $8 \mathrm{msec}$.

A number of observations suggest an active size tradeoff between R1 and R2. First, augmentation of R1 occurred at the expense of CR2 augmentation, which was close to control amplitude in all conditions. In contrast, in Experiments 1 and 2 , in which R1 was unaffected by the stimulus conditions, acoustic stimuli presented in the R1 latency at SOAs similar to those in the present experiment augmented CR2 amplitude. Second, percutaneous stimuli presented 3 and 9 msec after a leading percutaneous stimulus below threshold for R1 elicitation augment CR2 (Plant \& Hammond, 1989). Third, Sanes and Ison (1979) found that a leading acoustic stimulus that augmented R1 typically suppressed CR2 amplitude. An inverse relationship between $R 1$ size and CR2 size might result from initiation of eyelid closure by R1. An R2 that followed an R1 large enough to move the lid might then be smaller than otherwise to complete lid closure. Adaptive compensations of R2 size of this sort have been shown by Evinger and Manning (1988), who found that $R 2$ amplitude was reduced when their subjects wore small adapting weights that acted to close the lid, and thereby reduce the muscle contraction required for lid closure. Contrary to the earlier opinion that R2 alone was capable of causing lid movement (e.g., Shahani \& Young, 1972), more recent observations show that R1 does begin slow movement of the upper lid (Sunohara, Tomi, Satoyoshi, \& Tachibana, 1985).

\section{REFERENCES}

Blumenthal, T. D., \& Berg, W. K. (1986). The startle response as an indicator of temporal summation. Perception \& Psychophysics, 40, 62-68.

D'Aмато, M. R. (1979). Experimental psychology: Methodology, psychophysics, and learning. New Delhi: Tata McGraw-Hill.

Evinger, C., \& MANNING, K. A. (1988). A model system for motor learning: Adaptive gain control of the blink reflex. Experimental Brain Research, 70, 527-538.
Hammond, G. R., \& Leitner, D. L. (1990). Augmentation of the rat's acoustic startle reflex by non-reflexogenic stimuli. Behavioral Neuroscience, 104, 841-848.

Hays, W. L. (1963). Statistics for psychologists. New York: Holt, Rinehart and Winston.

Henneman, E. (1974). Spinal reflexes and the control of movement. In V. B. Mountcastle (Ed.), Medical physiology (13th ed., Vol. 1, pp. 651-667). Saint Louis, MO: Mosby.

JEWETT, D. L., \& WLLISTON, J. S. (1971). Auditory-evoked far fields averaged from the scalp of humans. Brain, 94, 681-696.

Kimura, J. (1973). The blink reflex as a test for brain-stem and higher central nervous system function. In J. E. Desmedt (Ed.), New developments in electromyography and clinical neurophysiology (Vol. 3, pp. 682-691). Basel: Karger.

Marsh, R., Hoffman, H. S., \& StITt, C. L. (1973). Temporal integration in the acoustic startle reflex of the rat. Joumal of Comparative \& Physiological Psychology, 82, 507-511.

ONGERBoer DE VisSER, B. W., \& KuYPERS, H. G. J. M. (1978). Late blink reflex changes in lateral medullary lesions: An electrophysiological and neuroanatomical study of Wallenberg's syndrome. Brain, 101, 285-294.

Plant, Y., \&ammond, G. R. (1989). Temporal integration of acoustic and cutaneous stimuli shown in the blink reflex. Perception \& Psychophysics, 45, 258-264.

SANES, J. N., ISON, J. R. (1979). Conditioning auditory stimuli and the cutaneous eyeblink reflex in humans: Differential effects according to oligosynaptic or polysynaptic pathways. Electroencephalography \& Clinical Neurophysiology, 47, 546-555.

Sazbon, L., Solzi, P., Steinvil, Y., * Becker, E. (1988). Blink reflex in patients in prolonged comatose state. Electromyography \& Clinical Neurophysiology, 28, 151-158.

SCHARF, B., BuUs, S. (1986). Audition I. In K. R. Boff, L. Kaufman, \& J. P. Thomas (Eds.), Handbook of perception and human performance: Vol. 1. Sensory processes and perception (pp. 14.1-14.71). New York: Wiley.

SChARF, B., HoutSMA, A. J. M. (1986). Audition II. In K. R. Boff, L. Kaufman, \& J. P. Thomas (Eds.), Handbook of perception and human performance: Vol. 1. Sensory processes and perception (pp. 15.1-15.60). New York: Wiley.

Schmalohr, D., \& LiNKE, D. B. (1988). The blink reflex in cerebral coma: Correlations to clinical findings and outcome. Electromyography \& Clinical Neurophysiology, 28, 233-244.

Shahani, B. T., \& Young, R. R. (1972). Human orbicularis oculi reflexes. Neurology, 22, 149-154.

Sherrick, C. E., \& Cholewiak, R. W. (1986). Cutaneous sensitivity. In K. R. Boff, L. Kaufman, \& J. P. Thomas (Eds.), Handbook of perception and human performance: Vol. I. Sensory processes and perception (pp. 12.1-15.58). New York: Wiley.

Sunohara, N., Tomi, H., Satoyoshi, E., Tachibana, S. (1985). Glabella tap sign: Is it due to a lack of R2-habituation? Journal of the Neurological Sciences, 70, 257-267.

(Manuscript received August 7, 1990; revision accepted for publication December 17, 1990.) 